\title{
Parallel Computing of Patch-Based Nonlocal Operator and Its Application in Compressed Sensing MRI
}

\author{
Qiyue Li, ${ }^{1}$ Xiaobo Qu, ${ }^{1}$ Yunsong Liu, ${ }^{1}$ Di Guo, ${ }^{2}$ Jing Ye, ${ }^{1}$ Zhifang Zhan, ${ }^{1}$ and Zhong Chen ${ }^{1}$ \\ ${ }^{1}$ Departments of Communication Engineering and Electronic Science, Fujian Provincial Key Laboratory of Plasma and Magnetic \\ Resonance Research, Xiamen University, Xiamen 361005, China \\ ${ }^{2}$ School of Computer and Information Engineering, Xiamen University of Technology, Xiamen 361024, China
}

Correspondence should be addressed to Xiaobo Qu; quxiaobo@xmu.edu.cn and Zhong Chen; chenz@xmu.edu.cn

Received 1 April 2014; Accepted 27 April 2014; Published 20 May 2014

Academic Editor: Peng Feng

Copyright (C) 2014 Qiyue Li et al. This is an open access article distributed under the Creative Commons Attribution License, which permits unrestricted use, distribution, and reproduction in any medium, provided the original work is properly cited.

\begin{abstract}
Magnetic resonance imaging has been benefited from compressed sensing in improving imaging speed. But the computation time of compressed sensing magnetic resonance imaging (CS-MRI) is relatively long due to its iterative reconstruction process. Recently, a patch-based nonlocal operator (PANO) has been applied in CS-MRI to significantly reduce the reconstruction error by making use of self-similarity in images. But the two major steps in PANO, learning similarities and performing 3D wavelet transform, require extensive computations. In this paper, a parallel architecture based on multicore processors is proposed to accelerate computations of PANO. Simulation results demonstrate that the acceleration factor approaches the number of CPU cores and overall PANO-based CS-MRI reconstruction can be accomplished in several seconds.
\end{abstract}

\section{Introduction}

The slow imaging speed is one of major concerns in magnetic resonance imaging (MRI). Compressed sensing MRI (CSMRI) has shown the ability to effectively reduce the imaging time. Numerous researches have been conducted on CS-MRI [1-4] in the past few years. As one key assumption of CS-MRI, MR images are sparse in some transform domains $[5,6]$ and the sparsity seriously affects the reconstructed images. Unlike using predefined basis, such as wavelets, to represent MRI images, self-similarity of an MR image has been introduced into CS-MRI [7-10] with the help of nonlocal means (NLM) [11] or block matching and 3D filtering [12]. By modeling the similar patches embedded in MR images with a linear representation, a patch-based nonlocal operator (PANO) [13] has been shown to outperform typical total variation, redundant wavelets, and some other methods. PANO can be viewed as an alternative form of the block matching 3D frames [14]. It explores the similarities of nonlocal image patches and allows integrating conventional transforms such as wavelet, discrete cosine wavelet, or discrete Fourier transform to further exploit the sparsity of grouped similar image patches.
However, PANO usually suffers from massive computations because of high overlapping among the patches. Fortunately, learning patch similarities and forward and backward transformations among grouped patches are independent of each other. This allows reducing the reconstruction time by taking the advantage of the technology of parallel computing $[15,16]$. In this paper, we design a parallel architecture to accelerate the computations of PANO. There are two main steps in PANO [13], learning similarities and performing 3D wavelet transform on grouped patches. Simulation results demonstrate that the parallel architecture can effectively accelerate the computation speed by making use of multiple CPU cores. This parallel architecture is also widely applicable to the self-similarity-based image reconstruction methods.

\section{Methods}

As mentioned above, there are two major steps in PANO, learning similarities and performing $3 \mathrm{D}$ wavelet transform on grouped patches. 
We first review the process of learning similarities. For a specified patch, PANO is to find Q-1 similar patches (the shorter the Euclidean distance is, the more similar they are) in a search region centered in the specified patch [13]. After the similarity information of a patch is learnt, the $Q$ patches (including the specified patch and Q-1 similar patches) will be stacked into a $3 \mathrm{D}$ cube from the most similar one to the least, as is shown in Figure 1.

As $3 \mathrm{D}$ cubes are created, 3D wavelet transform can be applied to them. First, perform 2D forward wavelet transform in $\mathrm{X}-\mathrm{Y}$ dimension and then do $1 \mathrm{D}$ forward wavelet transform in $\mathrm{Z}$ dimension. After the forward wavelet transform is done, a soft threshold operator is applied. Following 3D backward wavelet transform, 3D cubes are assembled back to the image.

The two major steps in PANO are both patch-based, and each patch can be handled independently. Therefore, parallel computing can be a good solution to accelerate the computations. More and more image processing applications that exhibit a high degree of parallelism are showing great interests in parallel computing [16]. Parallelization can be easily implemented on multicore central processing unit (CPU) with existing application program interfaces (APIs) such as POSIX threads and OpenMP [15]. Meanwhile, many applications are making use of graphic processing unit (GPU) [17], which is good at doing complex computations. Due to the simplicity, flexible interface, and multiplatform supports, we choose OpenMP as our API to develop parallel computing program on $\mathrm{CPU}$, which is the common configuration for personal computers.

2.1. Parallelization on Learning Similarities. In order to make full use of a multicore CPU, tasks should be properly generated and assigned to CPU cores [16]. If there are too many tasks, CPU cores will switch frequently to handle the tasks and the cost for switching between CPU cores is expensive. Therefore, generating a task that is based on a single patch is suboptimal. Instead, generating a task that processes a serial of image patches in one column or one row can be better, as is shown in Figure 2. Because generating tasks according to columns is the same as according to rows, we choose columns as an example in this paper. Computations in one task are serial while each task will be processed in parallel. After one task is done, computations on the patches of the corresponding column will be finished. That is, all the similarity information for the patches in that column is learnt. CPU cores can be effectively utilized when the number of columns in an image is much larger than the number of CPU cores in a personal computer.

2.2. Parallelization on $3 D$ Wavelet Transform on Cubes. After similarity information is learnt, 3D cubes can be easily created, as is shown in Figure 1. The 3D wavelet transform on cubes shares the same parallel architecture as Section 2.1. One task gets out the similarity information of patches in one column. After cubes are created according to the learnt similarity information, wavelet transforms will be applied on them. Computations on cubes within each column of patches will be serial and tasks will be processed in parallel, as is shown in Figure 3.

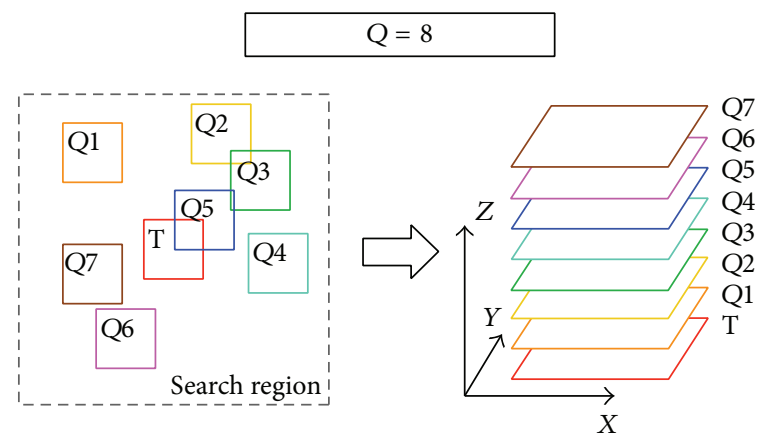

FIGURE 1: Search Q-1 = 7 similar patches in the search region and stack them with the basic patch $\mathrm{T}$ into a $3 \mathrm{D}$ cube.

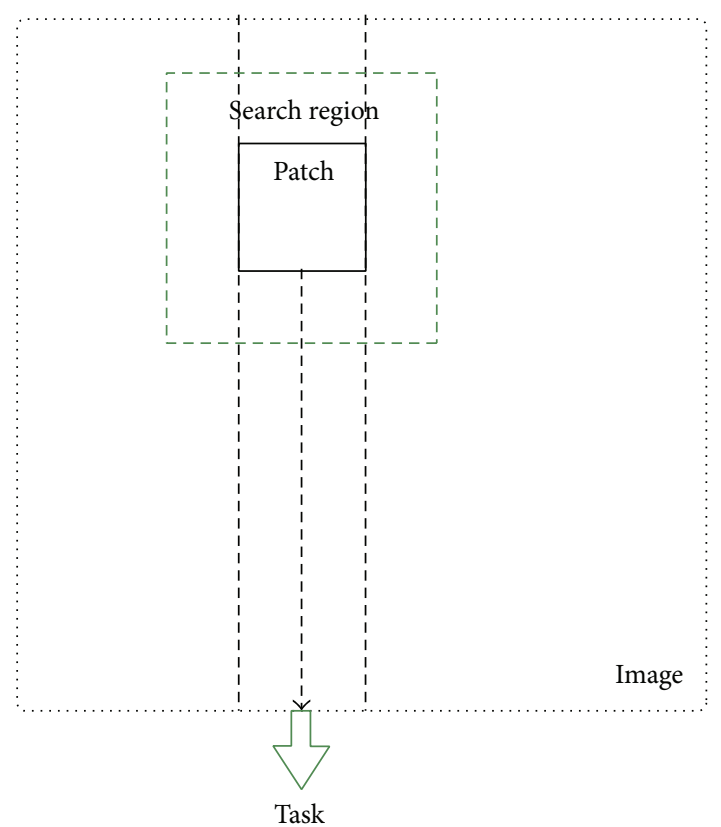

FIgURE 2: Generating tasks according to image columns. One task corresponds to one column of patches.

\section{Results}

Experiments are conducted in three aspects. First, the effectiveness of the parallel architecture on a personal computer is discussed. Second, the performance on a professional workstation with $100 \mathrm{CPU}$ cores will be explored. At last, a complete process in PANO-based CS-MRI will be accomplished to see how much the improvement will be in iterative CS-MRI reconstructions. Every experiment is repeated five times, and the reported computation time is the average of them.

3.1. Parallelization Improvement on a Personal Computer. The experiments are conducted on DELL T1700 personal workstation with E3-1225v3 CPU (4 cores, 3.2 GHz). 64-bit Windows 7 is our operating system. MATLAB version is 2013B with MEX compiler from Visual Studio 2012. Patch size is set to be $8 \times 8$, search region is $39 \times 39$, sliding 


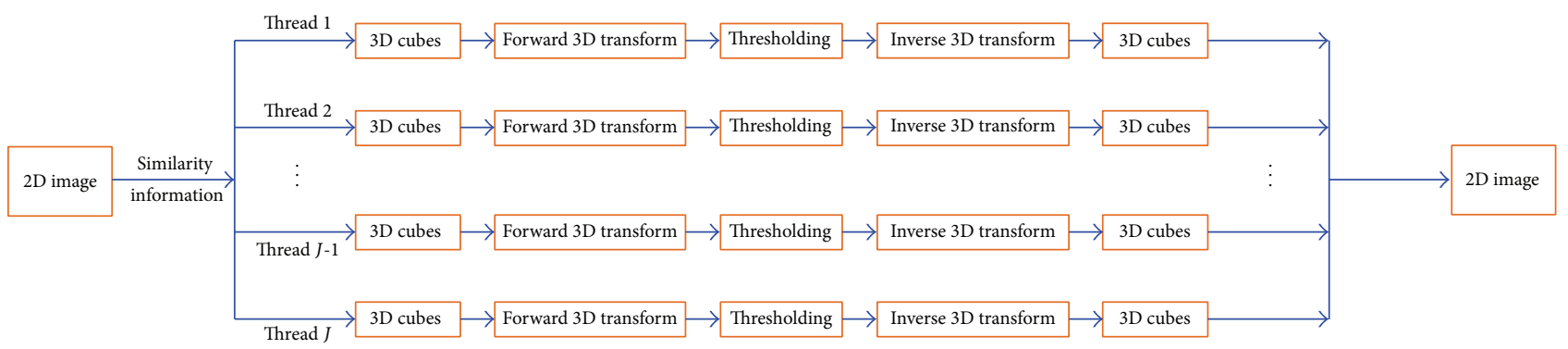

Figure 3: Parallel architecture of 3D wavelet transform on cubes.

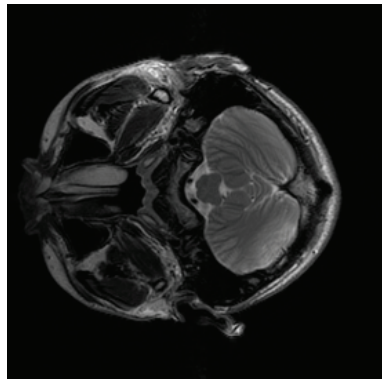

(a)

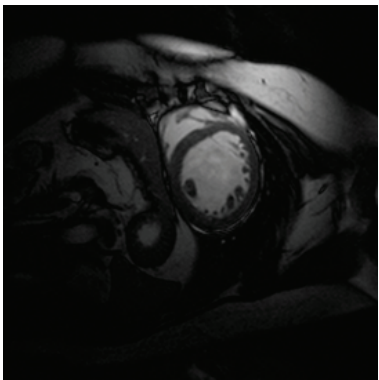

(b)

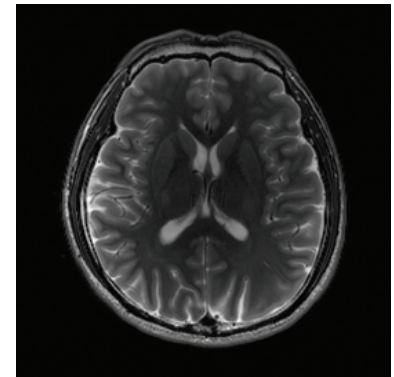

(c)

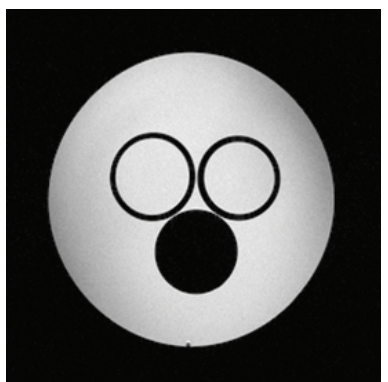

(d)

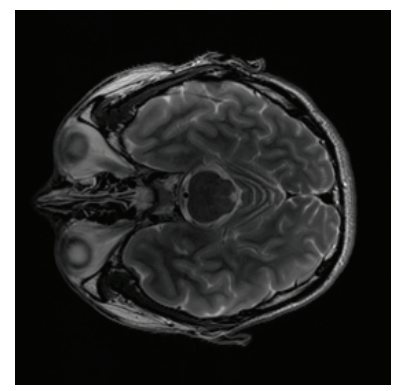

(e)

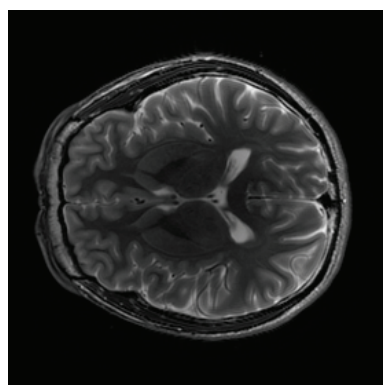

(f)

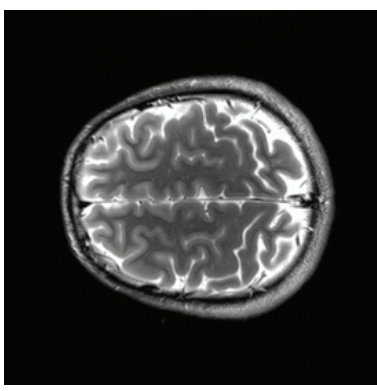

(g)

Figure 4: Dataset. (a), (c), (e), (f), and (g) T2 weighted MR brain images, (b) one frame of cardiac MR images, and (d) a water phantom image.

step is 1 , and the number $Q$ is 8 . Sliding step is a moving step to select the next patch. Haar wavelet is chosen as the sparsifying transform due to its efficiency and simplicity. Seven images shown in Figure 4 are tested. Figures 4(a), 4(c), $4(\mathrm{e}), 4(\mathrm{f})$, and $4(\mathrm{~g})$ are T2 weighted brain images acquired from a healthy volunteer at a $3 \mathrm{~T}$ Siemens Trio Tim MRI scanner using the T2 weighted turbo spin echo sequence $(\mathrm{TR} / \mathrm{TE}=6100 / 99 \mathrm{~ms}, 220 \times 220 \mathrm{~mm}$ field of view, and $3 \mathrm{~mm}$ slice thickness). Figure 4(b) is a cardiac image downloaded from Bio Imaging Signal Processing Lab [18, 19]. Figure 4(d) is a water phantom image acquired at 7T Varian MRI system (Varian, Palo Alto, CA, USA) with the spin echo sequence $(\mathrm{TR} / \mathrm{TE}=2000 / 100 \mathrm{~ms}, 80 \times 80 \mathrm{~mm}$ field of view, and $2 \mathrm{~mm}$ slice thickness).

3.1.1. Accelerating in Learning Similarities. As is shown in Figure 5, computation time for learning similarities of Figure 4(a) is effectively decreased with the increasing number of CPU cores in use. It demonstrates that the parallelization is effective. However, the accelerating factor cannot be exactly the same as the number of CPU cores. One reason is that it needs to create and assign tasks to CPU cores, and this activity requires extra time. Furthermore, tasks may not be finished exactly within the same time. Therefore, it is reasonable that the accelerating factor is between 3 and 4 with 4 CPU cores.

3.1.2. Accelerating in 3D Wavelet Transform on Cubes. As is shown in Figure 6, parallelization on 3D wavelet transform on cubes of Figure 4(a) is also very effective. Besides the cost introduced in Section 3.1.1 for parallelization, it also needs time to create cubes and reassemble them back to the image. Therefore the accelerating factor is smaller than four when there are $4 \mathrm{CPU}$ cores in use.

3.1.3. Test Results on More MRI Images. The remaining images in Figure 4 are tested and the computation time is shown in Figure 7. Because Figures 4(b)-4(g) are complex images, the computation time of $3 \mathrm{D}$ wavelet transform 


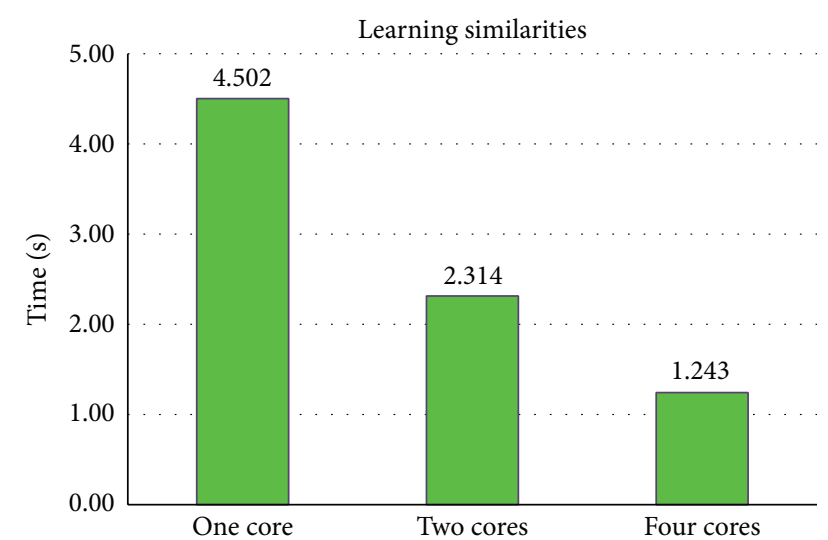

FIGURE 5: Computation time of learning similarities for Figure 4(a) with different number of CPU cores in use.

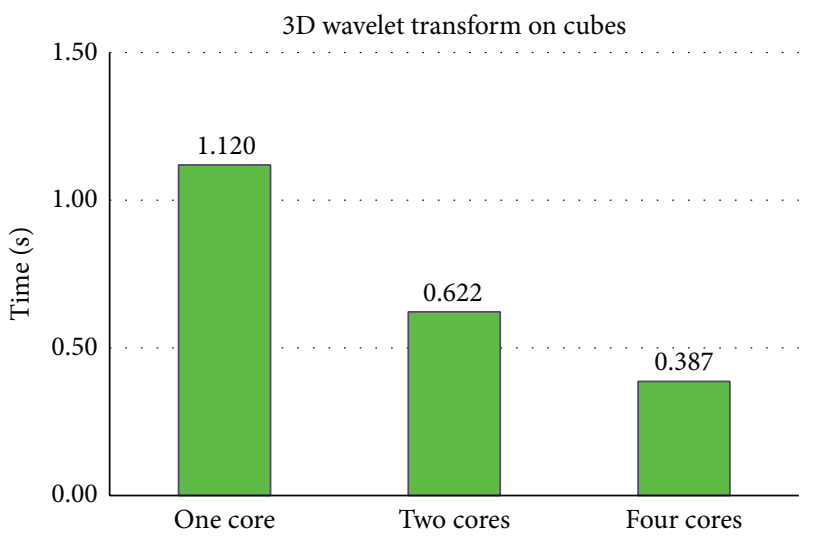

FIGURE 6: Computation time of 3D wavelet transform on cubes for Figure 4(a) with different number of CPU cores in use.

on cubes is approximately twice the consuming time for Figure 4(a), as real and imaginary parts are computed separately. The computation time is comparable for different images, implying that the computations do not depend on the image structures. Furthermore, as it can be seen in Figures $7(\mathrm{a}), 7(\mathrm{~b})$, and $7(\mathrm{c})$, the acceleration is effective with different images when testing with various CPU cores.

3.2. Parallelization Improvement on a Professional Workstation. The effectiveness on a professional workstation is discussed in this section. Experiments are executed on IBM x3850 with ten E7-8870 CPUs. As shown in Figures 8 and 9, both learning similarities and 3D wavelet transform on cubes are effectively accelerated with 100 cores, and 128 threads are tested due to hyperthreading technology [20].

\subsection{Parallelization Improvement on PANO-Based CS-MRI.} The full reconstruction of PANO-based CS-MRI is discussed in this section. Experiments conducted with the sliding steps are 8 and 4, respectively, and $40 \%$ of data are sampled. With a typical setting, similarity is learnt twice, and $3 \mathrm{D}$ wavelet transform on cubes will be called about 160 times. As introduced in [13, Algorithm 2], setting the times of updating

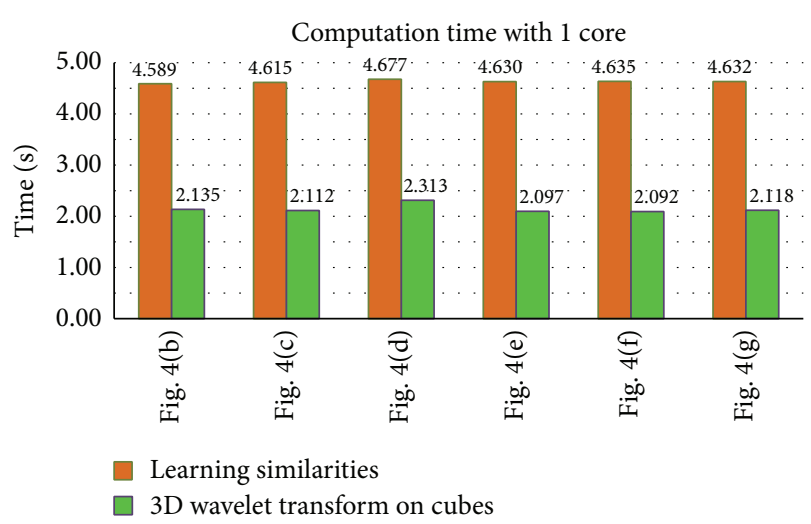

(a)

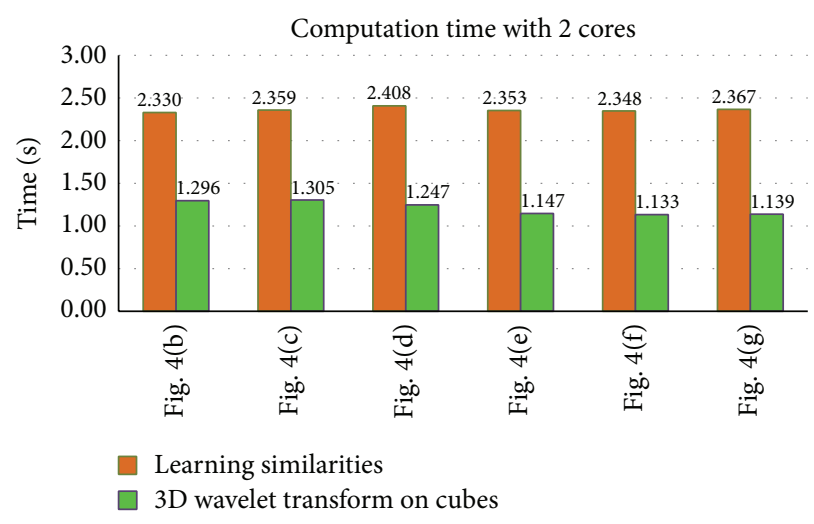

(b)

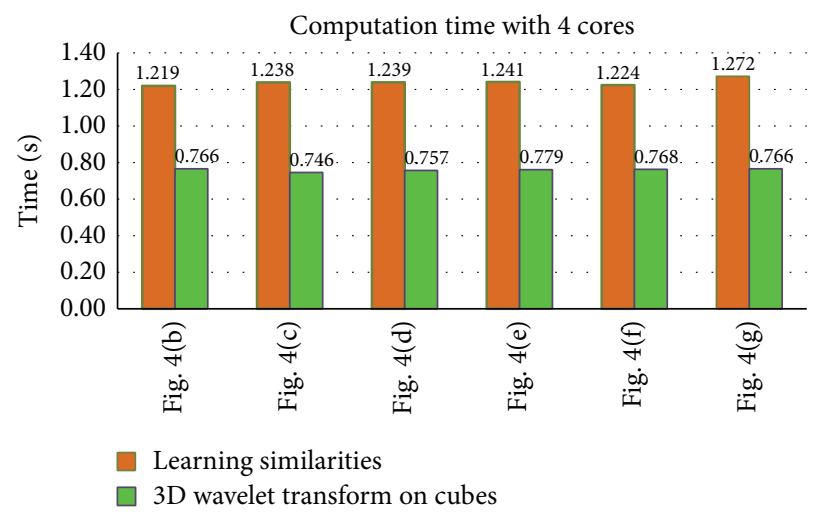

(c)

FIGURE 7: Computation time with various cores for (b), (c), (d), (e), (f), and (g) in Figure 4.

the guide image as 2 will improve results. Therefore similarity is learnt twice. In [13, Algorithm 1], the assembled image needs to be updated many times to get a sufficient large $\beta$. Besides, the 3D wavelet transform performed on cubes will be called about 160 times.

As is shown in Table 1, both learning similarities and 3D wavelet transform on cubes are accelerated effectively nearly 4 times with sliding step being 4 and 4 CPU cores in use. When sliding step is 8 , a full reconstruction can be accomplished in about 3 seconds. Because computations are very fast and 
TABLE 1: Computation time of PANO-based CS-MRI (unit: seconds).

\begin{tabular}{lcccc}
\hline & \multicolumn{2}{c}{ Sliding step $=8$} & \multicolumn{2}{c}{ Sliding step $=4$} \\
& One core & Four cores & One core & 0.578 \\
Learning similarities & 0.077 & 0.039 & 31.684 & 0.153 \\
3D wavelet transform on cubes & 7.910 & 2.438 & 0.560 & 8.915 \\
Other computations & 0.531 & 0.546 & $\mathbf{3 2 . 8 2 2}$ & 0.575 \\
Total reconstruction time & $\mathbf{8 . 5 1 8}$ & $\mathbf{3 . 0 2 3}$ & $\mathbf{9 . 6 4 3}$ \\
\hline
\end{tabular}

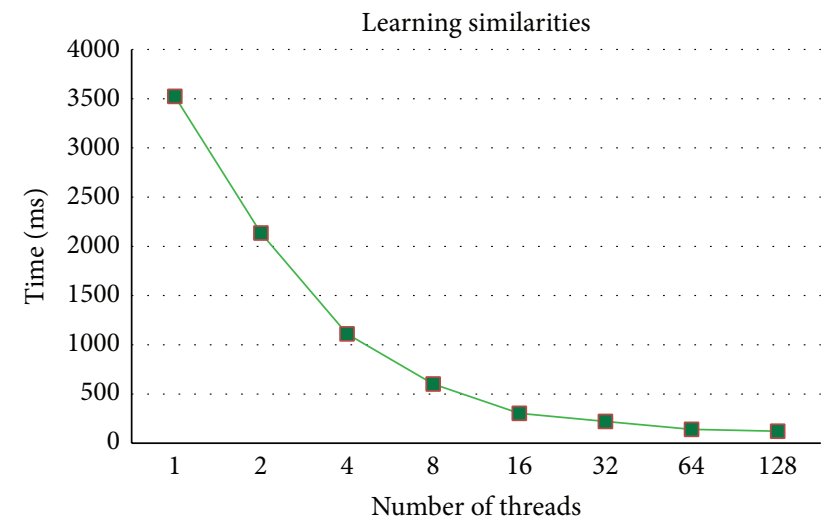

FIGURE 8: Computation time of learning similarities on a professional workstation for Figure 4(a).

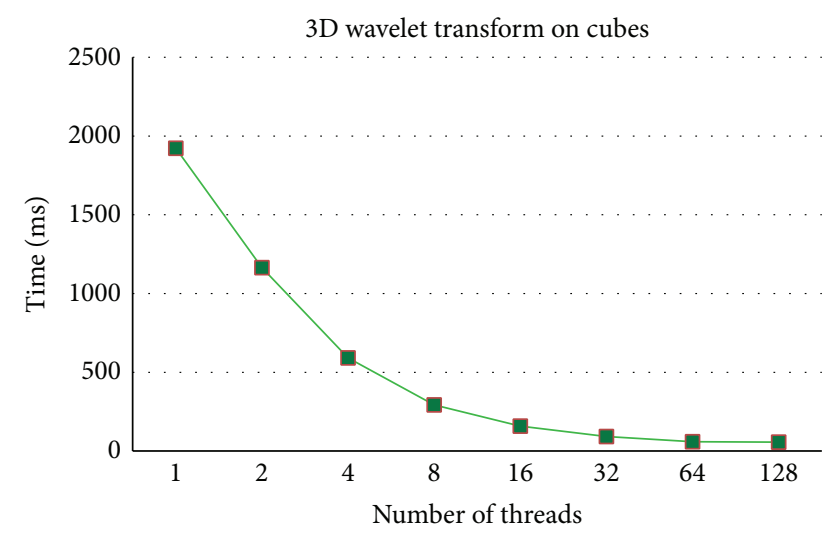

Figure 9: Computation time of 3D wavelet transform on cubes on a professional workstation for Figure 4(a).

the cost for parallelization is relatively expensive, learning similarities are accelerated less than 3 times.

\section{Conclusion}

In this paper, the patch-based nonlocal operator (PANO) has been parallelized to accelerate its two major steps, learning similarities and performing 3D wavelet transform on grouped similar patches. Experiments conducted on both the personal and professional computers have proven the effectiveness and applicability of the parallel architecture. Results demonstrate that a full PANO-based compressed sensing MRI reconstruction can be accomplished in several seconds. The parallel architecture of PANO is also applicable for other image reconstruction problems [21]. In the future, how to maximize the acceleration for computations of PANO on $3 \mathrm{D}$ imaging with multicore CPUs and GPUs will be developed.

\section{Conflict of Interests}

The authors declare that there is no conflict of interests regarding the publication of this paper.

\section{Acknowledgments}

The authors would like to thank Dr. Bingwen Zheng and Mr. Qianjing Shen for the valuable discussions. The authors are grateful to Dr. Michael Lustig for sharing spare MRI code and Dr. Jong Chul Ye for sharing cardiac data used in Figure 4(b). This work was partially supported by the NNSF of China (61201045, 61302174, and 11375147), Fundamental Research Funds for the Central Universities (2013SH002), Scientific Research Foundation for the Introduction of Talent at Xiamen University of Technology (YKJ12021R), and Open Fund from Key Lab of Digital Signal and Image Processing of Guangdong Province (2013GDDSIPL-07 and 54600321).

\section{References}

[1] N. Cai, S. R. Wang, S.-S. Zhu, and D. Liang, "Accelerating dynamic cardiac MR imaging using structured sparse representation," Computational and Mathematical Methods in Medicine, vol. 2013, Article ID 160139, 8 pages, 2013.

[2] M. Lustig, D. L. Donoho, J. M. Santos, and J. M. Pauly, "Compressed sensing MRI," IEEE Signal Processing Magazine, vol. 25, no. 2, pp. 72-82, 2008.

[3] M. Lustig, D. Donoho, and J. M. Pauly, "Sparse MRI: the application of compressed sensing for rapid MR imaging," Magnetic Resonance in Medicine, vol. 58, no. 6, pp. 1182-1195, 2007.

[4] M. Chen, D. Mi, P. He, L. Deng, and B. Wei, "A CT reconstruction algorithm based on $\mathrm{L}_{1 / 2}$ regularization," Computational and Mathematical Methods in Medicine, vol. 2014, Article ID 862910, 8 pages, 2014.

[5] D. L. Donoho, "Compressed sensing," IEEE Transactions on Information Theory, vol. 52, no. 4, pp. 1289-1306, 2006.

[6] E. J. Candes, J. Romberg, and T. Tao, "Robust uncertainty principles: exact signal reconstruction from highly incomplete frequency information," IEEE Transactions on Information Theory, vol. 52, no. 2, pp. 489-509, 2006. 
[7] K. Lu, N. He, and L. Li, "Nonlocal means-based denoising for medical images," Computational and Mathematical Methods in Medicine, vol. 2012, Article ID 438617, 7 pages, 2012.

[8] M. Akçakaya, T. A. Basha, B. Goddu et al., "Low-dimensionalstructure self-learning and thresholding: regularization beyond compressed sensing for MRI Reconstruction," Magnetic Resonance in Medicine, vol. 66, no. 3, pp. 756-767, 2011.

[9] G. Adluru, T. Tasdizen, M. C. Schabel, and E. V. R. Dibella, "Reconstruction of 3D dynamic contrast-enhanced magnetic resonance imaging using nonlocal means," Journal of Magnetic Resonance Imaging, vol. 32, no. 5, pp. 1217-1227, 2010.

[10] K. Egiazarian, A. Foi, and V. Katkovnik, "Compressed sensing image reconstruction via recursive spatially adaptive filtering," in Proceedings of the 14th IEEE International Conference on Image Processing (ICIP '07), vol. 1, pp. 549-552, San Antonio, Tex, USA, September 2007.

[11] A. Buades, B. Coll, and J. M. Morel, "A review of image denoising algorithms, with a new one," Multiscale Modeling \& Simulation, vol. 4, no. 2, pp. 490-530, 2005.

[12] K. Dabov, A. Foi, V. Katkovnik, and K. Egiazarian, "Image denoising by sparse 3-D transform-domain collaborative filtering," IEEE Transactions on Image Processing, vol. 16, no. 8, pp. 2080-2095, 2007.

[13] X. Qu, Y. Hou, F. Lam, D. Guo, J. Zhong, and Z. Chen, "Magnetic resonance image reconstruction from undersampled measurements using a patch-based nonlocal operator," Medical Image Analysis, 2013.

[14] A. Danielyan, V. Katkovnik, and K. Egiazarian, "BM3D frames and variational image deblurring," IEEE Transactions on Image Processing, vol. 21, no. 4, pp. 1715-1728, 2012.

[15] R. Shams, P. Sadeghi, R. A. Kennedy, and R. I. Hartley, "A survey of medical image registration on multicore and the GPU," IEEE Signal Processing Magazine, vol. 27, no. 2, pp. 50-60, 2010.

[16] D. Kim, V. W. Lee, and Y.-K. Chen, "Image processing on multicore x86 architectures," IEEE Signal Processing Magazine, vol. 27, no. 2, pp. 97-107, 2010.

[17] S. S. Stone, J. P. Haldar, S. C. Tsao, W.-M. W. Hwu, B. P. Sutton, and Z.-P. Liang, "Accelerating advanced MRI reconstructions on GPUs," Journal of Parallel and Distributed Computing, vol. 68, no. 10, pp. 1307-1318, 2008.

[18] H. Jung, K. Sung, K. S. Nayak, E. Y. Kim, and J. C. Ye, "kt FOCUSS: a general compressed sensing framework for high resolution dynamic MRI," Magnetic Resonance in Medicine, vol. 61, no. 1, pp. 103-116, 2009.

[19] “Fig. 4(b)," http://bisp.kaist.ac.kr/ktFOCUSS.htm.

[20] “HT Technology," http://en.wikipedia.org/wiki/Hyper-Threading.

[21] D. Guo, X. Qu, X. Du, K. Wu, and X. Chen, "Salt and pepper noise removal with noise detection and a patch-based sparse representation," Advances in Multimedia, vol. 2014, Article ID 682747, 14 pages, 2014. 


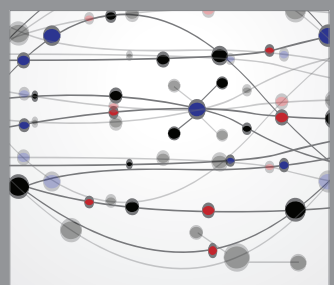

The Scientific World Journal
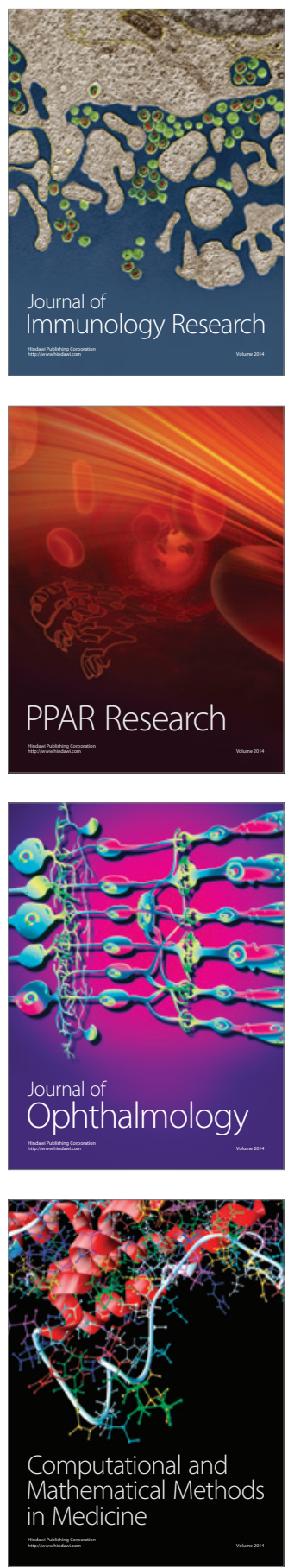

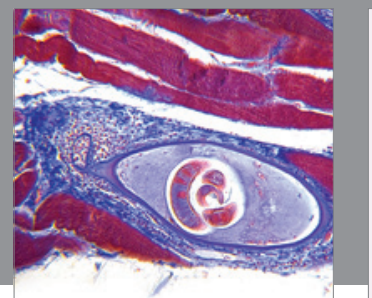

Gastroenterology

Research and Practice
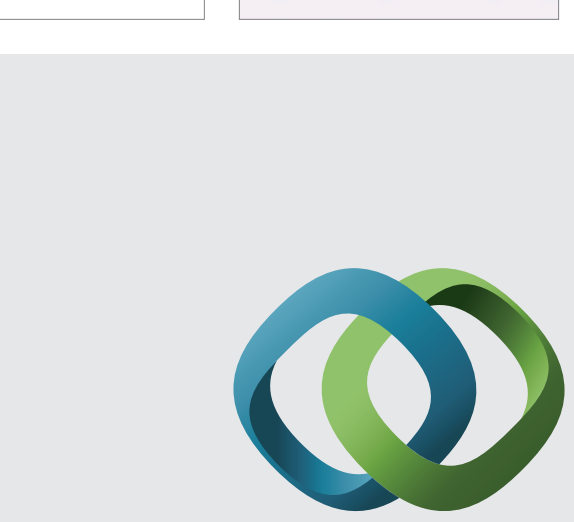

\section{Hindawi}

Submit your manuscripts at

http://www.hindawi.com
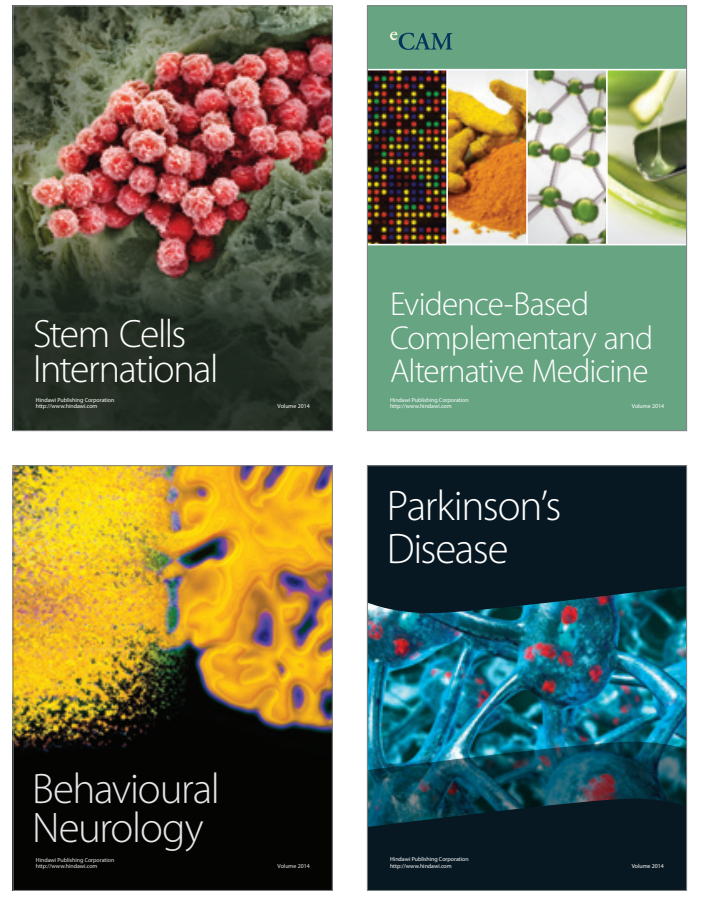
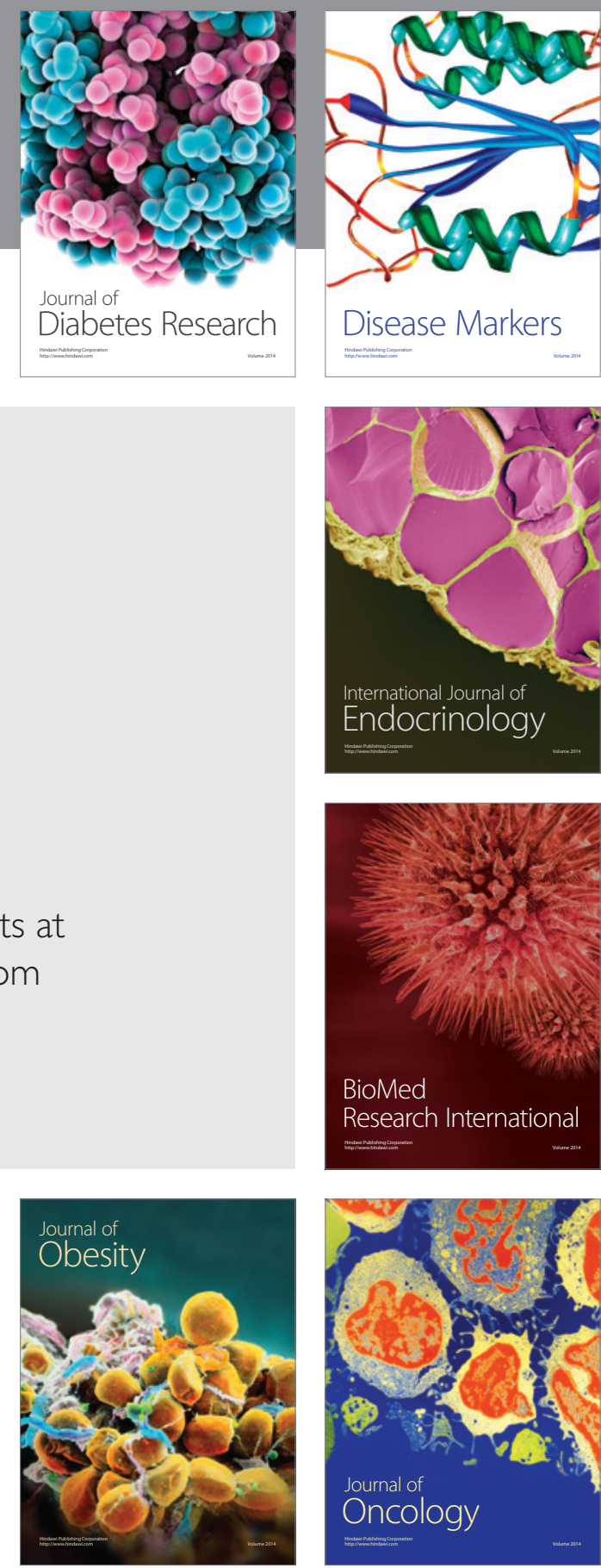

Disease Markers
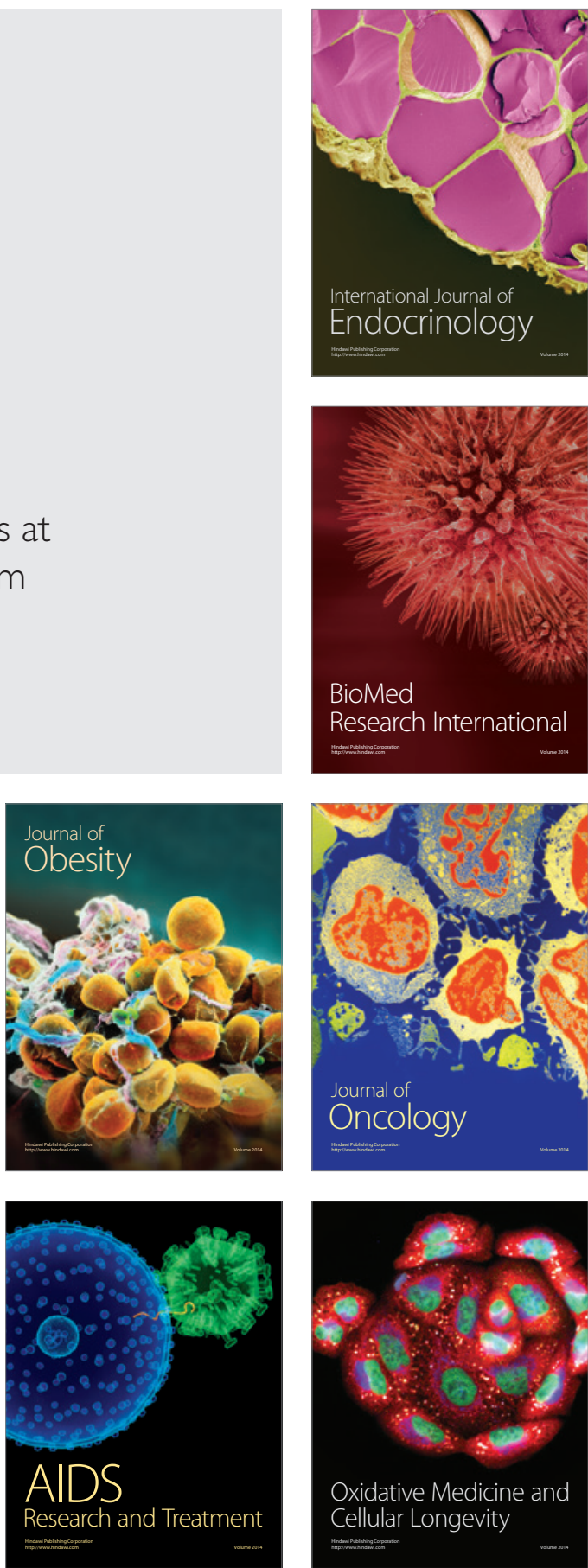